\title{
Study on Solving On-line Decision Making System of Power System Based on Big Data
}

\author{
Qi-yan DAl ${ }^{1}$ \\ ${ }^{1}$ Suzhou College of Information Technology, Suzhou, Jiangsu, China
}

Keywords: Big Data, Power System, Stability, Strategy, On-line decision making system

\begin{abstract}
The development of power big data technology provides the new opportunity for solving the problems in many technical fields of electric power system. This paper discusses the accuracy and implementation issues for solving on-line decision making system based on power big data. First, we elaborate the two existing technical contact and basic linking manner, emphasizing advantages on solving fast data analysis technology based on big data. In addition, we discuss technical difficulties and unable large-scale application of on-line decision making system. Finally, the application of electric big data that may bring new solutions to solve the on-line decision making system is illustrated. The study establishes the foundation for engineering applications of on-line decision making system.
\end{abstract}

\section{Introduction}

Rise of big data technology in various fields has attracted highest attention. "China Electric Power Big Data White Paper" [1] was published in 2013, which of this provide direction for the development of Chinese electrical power big data technology. Take this as an opportunity to try, the related technology research and application has becomes increasingly active. Universal big data platform is already in design and development. The characteristics of power big data can be summarized as $3 \mathrm{~V}$ and $3 \mathrm{E}$. $3 \mathrm{~V}$ means Volume, Velocity and Variety. 3E means Energy, Exchange and Empathy[2-6].The description of $3 \mathrm{~V}$ is close to description of other industry. The description of $3 \mathrm{~V}$ has a typical characteristics of power industry. It reflects the tremendous value big data apply in power system. Data means energy in short. by means of using big data analysis to achieve energy saving, security purposes[7,8].

Power electrical big data covers power generation, transmission, transformation, distribution, consumption and other aspects of electricity production and management. It's the inevitable process within the electric power industry technological innovation in energy revolution. It's not only technological advances, but also involved in power system management system, development philosophy, technical route and other major aspects of the revolutions. It is the leap of the value form of the next generation power in the era of big data.

The power grid is expanded in recent decades. It has become the world's largest power grid. The expanded power grid has brought great challenges to security and stability. In the traditional theoretical support three lines of defense of power system, Chinese power grid was no large-scale blackouts in recent decades. But three lines of defense of power system is also facing challenges, such as extreme operation mode of power grid. The second defense line is facing new challenges for not envisaged fault. For the strategies of the second defense line are made through off-line simulation and calculation. In some extreme operating mode of power system, The off-line strategies 
are difficultly thoughtful. This problems can be solved by on-line decision making system. But on-line strategies are facing constraints of speed and size of data calculation. Making online decision making system is difficult to play a practical role. The strategies of security and stability control devices are made by off-line simulation.

The development of power big data provides a new mentality and method for solving the problems that the on-line decision making strategies can't be practical. Through the fast speed calculation ability of big data, we can accurately calculate the online control strategies. The strategies executed by security and stability devices, which implemented accurately after the fault. It limits the fault influence in a small range.

\section{Smart Grid and Big Data}

The smart grid is highly integrated with information technology, computer technology, communication technology and the original transmission, distribution technology, to form a new power grid. It has many advantages, such as improving energy efficiency, minimizing environmental impacts, enhancing the power supply security, improving power supply reliability, reducing the energy loss consumption, etc. The concept of the smart grid getting more information on the users how to use electricity to optimize the production, distribution and consumption of electricity to make huge amounts of information interaction, to implement the information exchange between power grid equipment, and complete the information collection, measurement, control, protection, measurement and monitoring such as basic function based on modern network, communication and information technology. It can support grid real-time automatic control, intelligent adjustment, higher functions such as online decision making and collaborative interaction. Thus related researchers has pointed out that concept of the smart grid is the application of the big data in the power industry in an abstract way [9].

\section{Universal Framework of Big Data}

The general big data platform overall architecture of Apache open source technology foundation is shown in figure 3[10]. The architecture has good generality and is suitable for the electric power enterprise big data planning. Its main idea is based on distributed file processing system of Hadoop file system (the Hadoop distributed file system, HDFS) as a big data storage framework, distributed computing technology of MapReduce as big data processing framework. It makes the $\mathrm{PB}, \mathrm{ZB}$ level data query analysis to be possible based on the distributed computing technology. In addition the framework is also including business intelligence application, the traditional data warehouse, big data access framework, big data dispatching framework, network layer, operating systems, servers, backup and restore, and data management module, etc. 


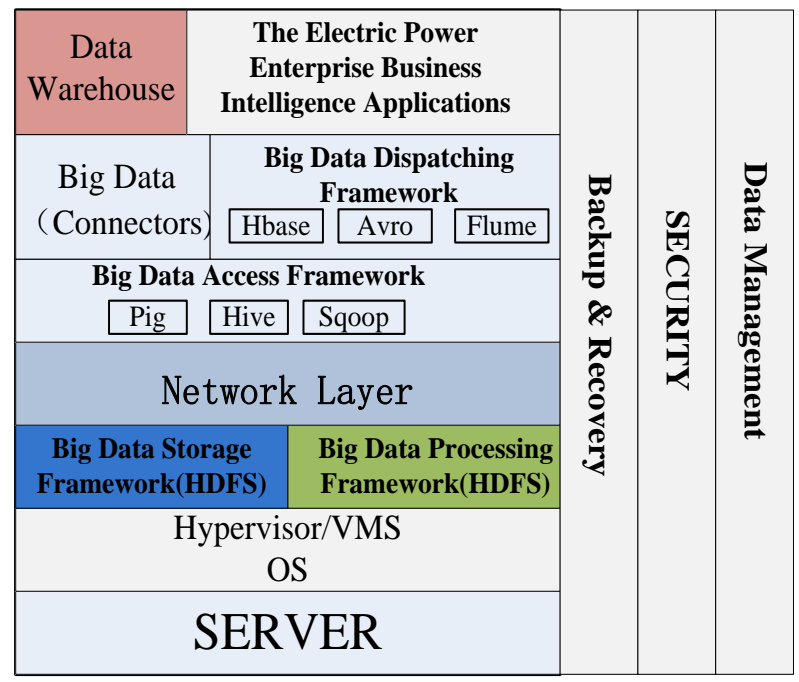

Figure 1. Universal framework of big data platform

The framework covers all area of big data technology. It is worth noting that we can not only access to distributed file storage system through this framework, but also can achieve access to the traditional data warehouse through big data connectors and open-source data transfer tools Sqoop.

\section{Role of Three Lines of Defense of Power System}

\section{Declaration of Three Lines of Defense of Power System}

The concept of three lines of defense is put forward by the specialists of the older generation engaging in electric power through a comprehensive summary of the lessons of Blackouts in China and around the world 60 to 70 years have occurred, and combined with the power system accident, the actual process of development and characteristics, which is targeted, vivid and practical, carrying out the principle of the Security and Stability Guideline for Power System. Except that necessary protective control measures should be taken, when power equipment is at fault, from the angle of accident prevention, fault shall be inspected from relay protection device of the equipment and faulty element shall be removed quickly. The quicker the faulty element is removed, the less the influence is made on power system. Generally, in a normal operational mode, for the single fault with much more probability to occur, the system is able to continuously maintain stable operation when the fault is eliminated. Therefore, we define tripping of the faulty elements as the first line of. For single serious fault or $\mathrm{N}-2$, $\mathrm{N}-3$ fault with less probability, the system may still have such problems as transient stability, overload of equipment or voltage stability when fault is eliminated. After the fault type and the operational mode prior to the fault are determined by stability control system, generator tripping shall be conducted in the sending end power plant of grid, and load shedding shall be conducted in receiving end grid and quick adjustment of direct current power of HVDC shall be conducted to ensure safe and stable operation of the system after the accidents. Therefore, stability control system and stability control measures are defined as the second line of defense of the grid. If the system is unable to keep synchronous step or has unstable voltage or frequency in case that a serious multiple fault occurs (for example, the breaker refuses to operate, protection device and stability control device refuse to operate or controlled quantity is inadequate, etc.). In this critical time, the grid should be split if the system is out-of-step, certain amount of 
load should be eliminated as soon as possible based on set value of low frequency and low voltage if frequency or voltage detected the significant deviates from the allowed value (the generator tripping in sending end system for high frequency) in order to let the remaining grid to regain power balance. So that it can prevent accidents from developing and prevent the system from the collapse and the large area outage. Therefore the third line of defense is comprised of out-of-step separation, load shedding based on low frequency and low voltage and high frequency generator tripping, which is also the last line of defense. If this line of defense is not effective, it is hard for the grid to avoid collapse. Once the system collapses, it will be forced to start up from black, which not only prolongs the period of power failure, but also results in great losses. Schematic diagram of the three lines of the defense is shown as figure 2[11].

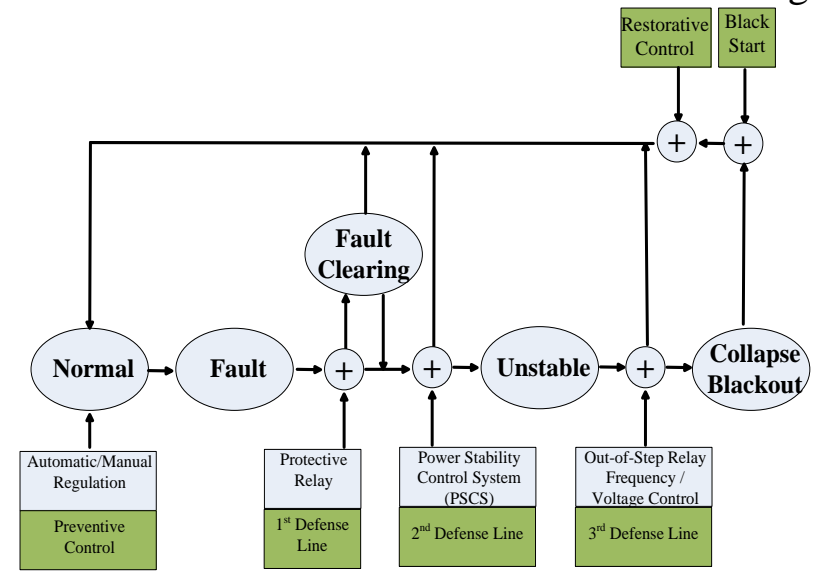

Figure 2. Schematic diagram for control phases of the three lines of defense of power system

To sum up, the three lines of defense are three protective walls setting against the grid faults to eliminate accidents at the beginning as much as possible and never let the accidents develop further. The concept of three lines of defense is clear and easy for understanding and operation. Each power giant in the world has their own provisions and measures to prevent the large area outage of power system, but they are not as clear and definite as the three lines of defense developed by China.

\section{Role of Online Decision-making System in Three Lines of Defense of Power System}

From the previous section, we must take effective control measures to keep the secure and stable operation of power grids before the power grid becomes instability, and the general fault mainly defense by the first line of defense of power system with the low probability of occurrence of a single serious fault or N, N-2, N-3 fault defense by the second line of defense of power system. The measures of second line of defense of power system are executed by security and stability control device.

The preparation methods of the control strategy sheets are divided into the off-line analysis and the on-line analysis. The off-line prepared strategy sheet is generally adopted at present, which has such disadvantages as large amount of calculation and maintenance and poor applicability for development of grid. On-line stability decision (figure 3) is the developing direction, but it needs to collect current operation conditions and data information of grid and calculate quickly and analyze algorithm and other special software as well as server platforms with high arithmetic speed. Currently it is applied in grid in Japan. However, generally the on-line decision is in the phase of engineering test, exploration and promotion. 


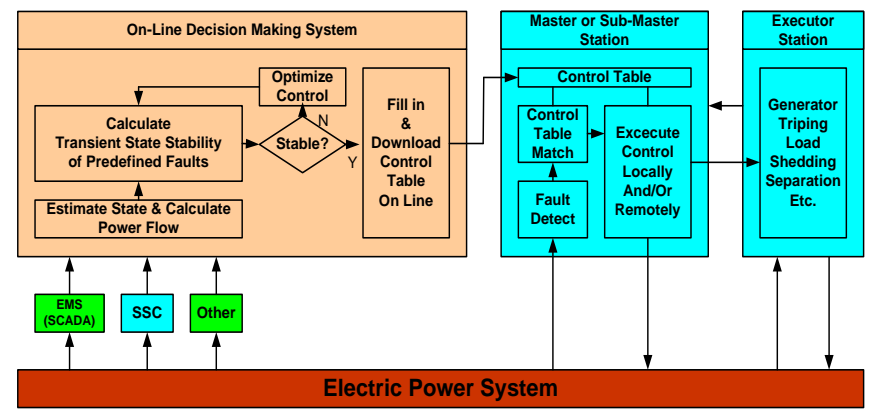

Figure 3. On-line decision, strategy update and implementation

It can be seen that although the online decision making system can adapt to flexible operation mode of power grid, but subject to the constraint of computing speed, computing server. So it's still not getting the practical application. In this regard, big data application is promising.

\section{Combination of Online Decision Making System and Big Data}

Figure 4 describes overall architecture of big data platform. It should combined with rich data flow in the power system to force the growth of specific industrial applications. Power system is a kind of high dimensional nonlinear complex system. Its internal data flow contains power flow, information flow, business flow, fault flow, meteorological flow and other different data. This paper mainly carries out research of solving the problems of on-line decision making system based on big data [12].

The graph below shows the combination universal framework of the power big data and on-line decision making system.

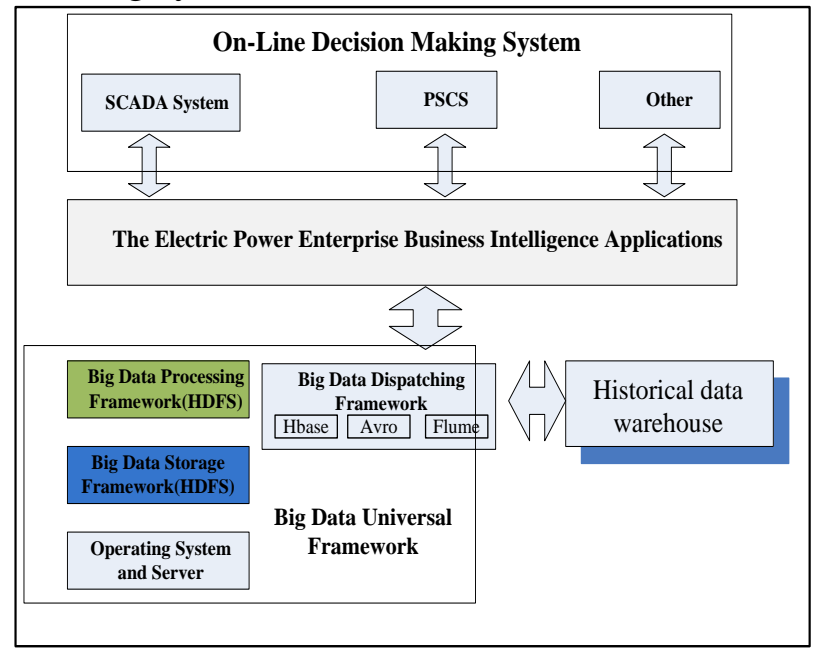

Figure 4. Link between universal framework of big data platform and On-line Decision Making System

Online decision making system is one source of information of power system. Through the combination with big data, we can clearly know power grid structure changes, the working state of each component, the change of electric quantity, and other information during the fault. We can know the real-time running state through the analysis of big data. According to the calculation of online decision making system, the stability control devices receive the strategies and execute to ensure that power system has corresponding stability control measures when happening extreme serious fault. To 
reduce the influence of faults, we have to guarantee the security and stability operation of power grid [13].

A typical online decision making system framework based on big data is shown in figure 5.The framework based on grid topology data, the operating data of grid and devices state, the algorithm model layer has judge index, optimization algorithm and evaluation index. We get library evaluation indexes through the calculation of evaluation indexes. On this basis, in the business layer through the weak area identification, causal tree analysis and evaluation index library, then joint judgment layer of library evaluation indexes, give the online decision control strategy. Finally through the execution layer making generator tripping, load shedding stability control measures.

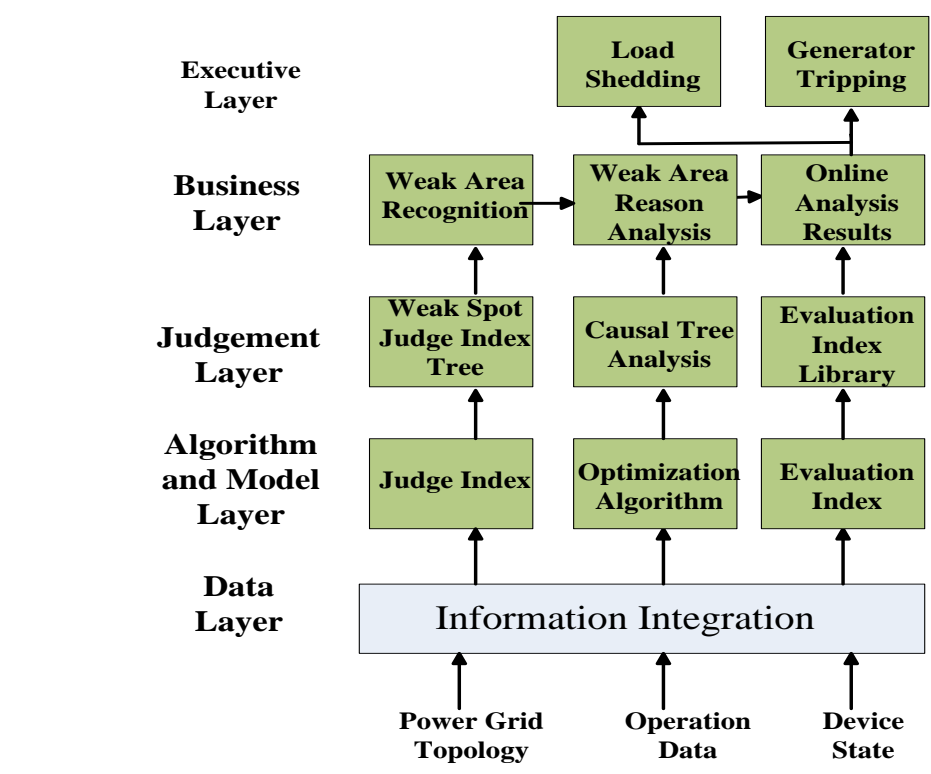

Figure 5. On-line Decision Making system Framework of Power System Based on Big Data

\section{Conclusion and Prospect}

As technology improves, big data will play an important role in the application of electric power industry. Big data may find new methods and thoughts to solve the problems which is difficult to be solved by traditional methods.

The paper discusses the relationship between smart grid and big data, and gives the overall executable framework of big data. It has the reference value for construction of electric power enterprises. In addition, this paper expounds the role of three lines of defense of power system. It explains the reasons why on-line decision making system cannot be engineering application. Combining the advantages of big data, analyzes the feasibility of big data applied to online stability control decision system. The on-line decision making system can make accurate decision to ensure the secure and stable operation of the power grid. Finally, the paper gives on-line decision making system framework of power system based on big data.

Big data applied in power system has just started. It has great significance to the development of power grid, combining technical advantage of big data and the demand of the power system. 


\section{References}

[1]Chinese society for electrical engineering informatization Committee. Chinese electric power big data development white paper(2013)[R]. Beijing: Chinese society for electrical engineering, 2013(in Chinese).

[2]Song Yaqi, Zhou Guoliang, Zhu Yongli. Present status and challenges of big data processing in smart grid[J]. Power System Technology, 2013, 37(4): 927-935(in Chinese).

[3]Qu Zhijian, Guo Liang, Chen Qiulin, et al.Intelligent dispatching lossless cluster compression technology based on Hadoop cloud framework[J]. Automation of Electric Power System, 2013, 37(18): 93-98(in Chinese).

[4]Qu Zhijian, Guo Liang, Chen Qiulin, et al. Intelligent dispatching lossless cluster compression technology based on Hadoop cloud framework[J]. Automation of Electric Power System, 2013, 37(18): 93-98(in Chinese).

[5]Huang Yanhao, YU Zhihong, Xie Chang,et al. Study on the Application of Electric Power Big Data Technology in Power System Simulation[J]. Proceedings of the CSEE, 2015, 35(1): 13-22.

[6]Yang Jinfeng, Liu Tao, Chen Qiguan, et al. Electric power customer online clustering based on mass measurement data[J]. East China Electric Power, 2013, 41(8): 1581-1585(in Chinese).

[7]Meng Xiaofeng, $\mathrm{Ci}$ Xiang. Big data management: Concepts, techniques and challenges[J]. Journal ofComputer Research and Development, 2013, 50(1): 146-169(in Chinese).

[8]Divyakant A, Philip B, Elisa B, et al. Challenges and opportunities with big data[J].Proceedings of the VLDB Endowment, 2012, 5(12): 2032-2033.

[9]Sun Bolin. Big data technology and its application in power industry[J]. Electric Age, 2013(8) : 18-23(in Chinese).

[10]Zhao Gang. Big data technology and application practice[M]. Beijing: Publishing House of Electronics Industry, 2013: 56-58(in Chinese).

[11]Techincal guide for eletric power system security and stability control. GB_T 26399-2011.

[12]Bai Jialin, Liu Tianqi, Cao Guoyun, et al. A survey onvulnerability assessment method for power system[J]. Power System Technology, 2008(S2): 26-30(in Chinese).

[13]com/smarterplanet/cn/zh/smart_grid/ideas/index.html. IBM. IBM smart grid solution(powergrid)[EB/OL](2014-09-20).http://www.ibm.com/smarterplanet/cn/zh/s mart_grid/ideas/index.html (in Chinese). 